\title{
Quantum Edge Extraction of Chest CT Image for the Detection and Differentiation of Infected Region of COVID-19 Patient
}

\author{
Rajib Chetia $^{1,2} \cdot$ Partha Pratim Sahu $^{1}$
}

Received: 4 March 2021 / Accepted: 5 October 2021

(c) King Fahd University of Petroleum \& Minerals 2021

\begin{abstract}
The COVID-19 outbreak requires urgent public health attention throughout the world due to having its fast human to human transmission. As per the guidelines of the World Health Organization, rapid testing, vaccination, and isolation are the only options to break the chain of COVID-19 infection. Lung computed tomography (CT) plays a prime role in the accurate detection of COVID-19. For detection and pattern analysis of COVID-19, here an improved Sobel quantum edge extraction with non-maximum suppression and adaptive threshold (ISQEENSAT) has been employed to extract clinical information of infected lungs suppressing minimal noises present in the chest. In comparison with conventional classical edge extraction operators, the proposed technique can detect more sharp and accurate clinical edges of peripheral ground-glass opacity that appeared in the initial stage of COVID-19 patients. The edge extraction results assure the detection and differentiation of COVID-19 infection. ISQEENSAT can be a useful tool for assisting COVID-19 analysis and can help the physician to detect the region how much it has infected.
\end{abstract}

Keywords COVID-19 - Quantum image processing $\cdot$ Chest CT image $\cdot$ Edge extraction

\section{Introduction}

The recent outburst of the severe acute respiratory syndrome (SARS) caused by COVID-19 has become one of the leading health concerns throughout the world. The COVID-19 has emerged in Wuhan city China, in December 2019 [1, 2], and later declared as a global pandemic by World Health Organization (WHO) due to faster human to human transmission in comparison with other human coronaviruses such as MERSCOV, SARS-COV, and alpha-COV [3, 4]. The SARS is developed mainly due to infection of the lungs with the novel corona virus. As per the guidelines of WHO, early detection of COVID-19 and control of transmission routes such as isolation and quarantine with treatment are the most effec-

Rajib Chetia

rajibofcit@gmail.com

Partha Pratim Sahu

ppstezu@gmail.com

1 Department of ECE, Tezpur University, Tezpur, Napam, Assam 784028, India

2 Department of ECE, Central Institute of Technology (CIT), Kokrajhar, BTAD, Assam 783370, India tive way to combat against COVID-19 outbreak [1]. There are many ways of screening for COVID-19 infection, such as real-time reverse transcription-polymerase chain reaction (rRT-PCR) [4], thermal scanning [5], antibody testing [6, 7], and abnormal respiratory patterns classifier [8]. Both thermal body scanners and respiratory patterns classifier are cheaper and faster screening of COVID-19 patients having no confirmation of COVID-19 infection. The rRT-PCR assures the testing of COVID-19 through gene expression analysis [4], but it is time-consuming, having chances of false results due to improper sampling and viral nucleic acid detection [9, 10]. Antibody testing is a rapid test, but it does not ensure the early-stage detection of infection due to the slow development of antibodies in the blood.

The SARS in COVID-19 patients is grown due to infection of lungs requiring continuous monitoring for the treatment of severe patients. In comparison with the above techniques, medical imaging such as chest X-ray, CT, and MRI imaging is noninvasive approach having less risk for its human-tohuman transmission during diagnosis. Chest $\mathrm{X}$-ray imaging is a commonly used technique for the diagnosis of infected lungs diseases. But severe COVID-19 patients require to take images repeatedly for continuous monitoring of the lungs, 
making it a high-risk factor due to damage of lung tissue with exposure of higher $\mathrm{X}$-ray doses in Chest $\mathrm{X}$-ray imaging than in CT scan imaging [11]. CT images provide 3D information about lung tissue conditions and provide more clinical information about infected lungs [11]. Moreover, chest CT imaging exhibits a speedy diagnosis of COVID- 19 with a low rate of failure. The magnetic resonance imaging (MRI) of lungs has poor image quality due to having low proton density in the lungs and rapid signal decay high magnetic susceptibility at the air-tissue interface. Further, it shows intrinsic motion artifacts because of respiratory and cardiac motion [12]. It is reported that chest CT scanning has more sensitivity than that of rRT- PCR due to improper clinical sampling and viral nucleic acid detection $[9,10]$. Chest $\mathrm{CT}$ can be useful as a standard method for the speedy diagnosis of COVID19 to improve the management of patients [13]. Conversely, a minute and detail clinical analysis of $\mathrm{CT}$ images is required to differentiate CT images of COVID-19 patients from other patients with viral pneumonia. The clinical features of CT images of COVID-19 patients are mainly ground glass opacity (GGO), crazy paving pattern (CPP), and consolidation that appeared in lungs [13]. The extraction of minute details of clinical features from CT images requires an image processing algorithm suppressing noises located in the chest. Machine learning and deep learning approaches have already been reported for COVID-19 diagnostic tools using chest Xray images $[14,15]$. But there has been no reduction in the complexity of time and space. Gaussian neuro-fuzzy multilayered data classifier has already been reported for early COVID prediction using Novel Corona Virus 2019 Dataset [16]. But this technique reveals no diagnosis of COVID-19 patients.

There are globally established classical edge extraction techniques, such as Sobel [17], Prewitt [18], Laplacian zero crossings [19], and Canny algorithms [20], which are used to detect the edges of an image. But the classical approach for edge extraction technique requires more bits to handle the processing during edge detection of huge data. In this regard, quantum image processing can play an important role in extracting clinical features from CT scan images. The quantum image processing technique consists of three main parts: image representation in terms of quantum images, quantum image processing with quantum computing, and lastly measuring the image from processed quantum images. Recently, quantum image processing based on quantum image representation has been used for the extraction of edges [21-23]. We have already studied an improved quantum Sobel operator [24] that extracts more edges of images in comparison with the classical Sobel operator [17] and other quantum operators $[21,25]$. But the main issue of this technique is that the extracted edges are not sharp [24]. Here, we propose an improved Sobel quantum edge extraction with non-maximum suppression and adaptive threshold
(ISQEENSAT) algorithm in Fig. 1. In the Sobel operator, the gradient estimation is limited to vertical and horizontal directions, making it hard to detect the edge information in the diagonal directions. The improved Sobel operator raises two masks for detecting diagonal edges. It detects more edges than conventional Sobel operator. Non-maximum suppression operation has the effect of removing all image information that does not form part of the local maxima. Consequently, edges will be sharper compared to the classical and quantum Sobel operators. The adaptive threshold method is used in our algorithm to set the threshold value because manually setting the threshold value is a random technique. The adaptive threshold value is determined from the onethird grayscale value of $3 \times 3$ neighborhood pixels window. Therefore, it avoids the detection of a false edge in the low threshold value and loss of information in the high threshold value since the threshold value is adapted. Our proposed algorithm can achieve more with sharper edge information that can be a suitable technique to extract clinical information of infected lungs of COVID-19 patients, suppressing minimal noises present in the chest $\mathrm{CT}$ images.

\subsection{Motivation}

The COVID-19 caused by the SARS-CoV-2 epidemic requires emergency public health care around the world. In many conventional operator-based edge extraction algorithms, false edge detection, noise sensitivity, the prediction accuracy of edges, and edges preservation in the diagonal direction are not improved. Recently, machine learning and deep learning approaches have been proposed for COVID19 diagnostic tools using radiography images [15]. However, the time and space complexity has not been minimized. In this direction, quantum image processing plays an important role in edge detection by considering the unique properties of quantum mechanics such as entanglements, superposition, and parallelism. The number of qubits (say $\mathrm{n}$ ) required to store information in the quantum representation is $2^{n}$ times less than classical image processing [22, 26]. For an $2^{n} \times$ $2^{n}$ image, no conventional edge extraction algorithm can complete the entire operation within the computational complexity of less than $O\left(2^{2 n}\right)$. That is the main reason why the classical edge extraction algorithms are inefficient for handling the sharp increase of huge data. Consequently, QIP algorithms are faster than conventional algorithms and significantly increase quantum storage capacity. The above properties of QIP and its utilization in image edge extraction techniques have motivated to development of quantum image processing algorithms for edge extraction of chest CT images for the detection and differentiation of infected regions of COVID-19 patients by minimizing the disadvantages of existing conventional and quantum edge extraction algorithms. The proposed improved Sobel operator-based 


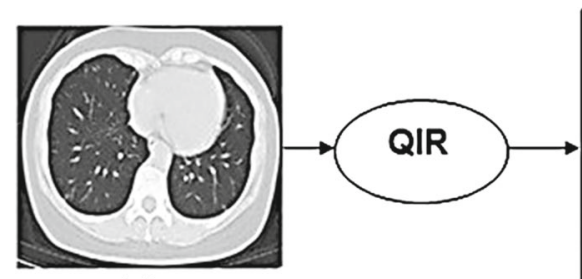

(a)

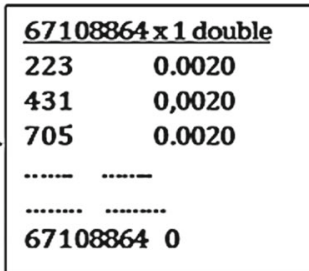

(b)

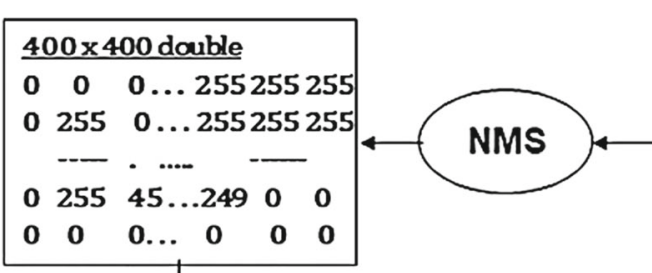

(e)
$400 \times 400$ double

255255255 ... 255255255

$255255244 \ldots 255255255$

$25525520 \ldots .17220255$

$255255255 \ldots 255255255$

\section{$400 \times 400 \mathrm{cell}$}

$256 \times 1 d \ldots . . .256 \times 1 d$

$256 \times 1 d \ldots . .256 \times 1 d$

$256 \times 1 d \ldots . . . . .256 \times 1 d$

$256 \times 1 d$......... $256 \times 1 d$

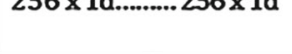

(c)

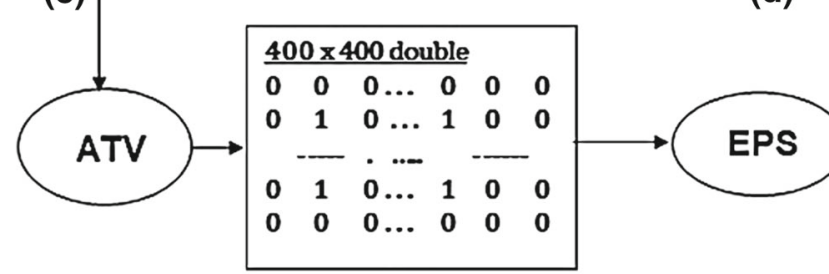

(f)

(d)

Fig. 1 Steps of pixels matrix representation of improved quantum edge extraction with non-maximum suppression and adaptive threshold (QIR—representation of quantum image, QIS—quantum image shifting, DPG — determination of pixel gradient, NMS — non-maximum suppression, ATV—adaptive threshold value, and EPS—edge point

quantum image edge extraction algorithm can extract more edge information, accurate edge location, and resolve realtime image processing problems.

\subsection{Contribution}

Contributions for this work are outlined below.

- An improved Sobel quantum edge extraction with nonmaximum suppression and adaptive threshold (ISQEENSAT) has been employed to extract clinical information of infected lungs suppressing minimal noises present in the chest. Different steps of the proposed ISQEENSAT algorithm and its related quantum states are introduced.

- To develop quantum code in MATLAB for the implementation of our proposed algorithm using MATLAB R2018a environment on a classical computer Intel (R) Core $^{\mathrm{TM}}$ 58300HCPU2.30 GHz, 8.00 GB RAM.

- To analyze the edges extracted from the clinical features of the chest CT scan images for a wide range of age groups.

- It has been observed that the proposed method has the highest PSNR, entropy, and SSIM in comparison with conventional methods. estimation). a Chest CT scan image of COVID-19 patient; b NEQR representation of image; $\mathbf{c}$ representation after QIS; $\mathbf{d}$ representation after DPG; e representation after NMS; $\mathbf{f}$ representation after ATV; g edge extracted image

- The circuit complexity of our algorithm is estimated as $\mathrm{O}$ $\left(n^{2}+q^{3}\right)$, which is lower than that of existing algorithms.

\section{Materials and Methods}

Our proposed algorithm consists mainly of six steps-representation of quantum image (QIR), quantum image shifting (QIS), determination of pixel gradient (DPG), non-maximum suppression (NMS), comparison with an adaptive threshold value (ATV), and edge point estimation (EPS). In step-1, the original chest CT scan image (Fig. 1a) is converted into a quantum image by using novel enhanced quantum representation (NEQR) technique (Supplementary S.1: Fig. S1) stored $3 \times 3$ neighboring pixel window (Fig. 1b). In step2 , the cyclic shift transformation is employed to derive the shifted quantum image set (shown in Fig. 1c) using $X$ shift and Y-shift transformation (supplementary S.2: Fig-S2). In step-3, the pixel gradients (Fig. 1d) are estimated by using horizontal, vertical, $45^{\circ}$, and $135^{\circ}$ directional Sobel masks (supplementary S.3: Fig-S.3.1(c)). In step-4, the nonmaximum suppression technique is used to determine the local maximum of the gradient image (Fig. 1e) (supplemen- 
tary S.4: Fig-S4). In step-5, we have used four quantum comparators to compare the gradient values with adaptive threshold values (Fig. 1f) (supplementary S.5: Fig-S5). Finally, in step-6, we have extracted edge pixels that indicate clinical information (supplementary S.6: Fig-S6). The output extracted image is shown in Fig. 1g.

Step-1 First, we convert the conventional image to quantum image by using NEQR method [27]. In NEQR technique,

$2 n+q$ qubits are needed to store $2^{n} \times 2^{n}$ size of an image. The quantum image $|I\rangle$ can be represented as follows:

$\left|I_{Y X}\right\rangle=\frac{1}{2^{n}} \sum_{Y=0}^{2^{n}-1} \sum_{X=0}^{2^{n}-1}\left|C_{Y X}\right\rangle|Y\rangle|X\rangle$

where grayscale value $C_{Y X} \in\left[0,2^{q}-1\right]$.

Step-2 Eight extra qubits are tensor product with quantum image $|I\rangle$ to store the $3 \times 3$ neighborhood pixels window and parallel controlled NOT (CNOT) operation is used to copy it, i.e.,

$$
\begin{aligned}
|0\rangle^{\otimes^{8 q}} \otimes\left|I_{Y X}\right\rangle & =\frac{1}{2^{n}} \sum_{Y=0}^{2^{n}-1} \sum_{X=0}^{2^{n}-1}|0\rangle^{\otimes^{8 q}}\left|C_{Y X}\right\rangle|Y\rangle|X\rangle \\
& =\frac{1}{2^{n}} \sum_{Y=0}^{2^{n}-1} \sum_{X=0}^{2^{n}-1}|0\rangle^{\otimes^{q}} \ldots|0\rangle^{\otimes^{q}}\left|C_{Y X}\right\rangle|Y\rangle|X\rangle
\end{aligned}
$$

In this step, cyclic shift transformations (Supplementary S.2) and CNOT operations (Supplementary S.3.3) are used on the quantum image $\left|I_{Y X}\right\rangle$ simultaneously. After these operations, any nine $3 \times 3$ neighborhood window of shifted quantum image sets are obtained and it can be represented as

$$
\begin{aligned}
& \left|C_{Y+1, X+1}\right\rangle,\left|C_{Y+1, X}\right\rangle,\left|C_{Y+1, X-1}\right\rangle, \\
& \left|C_{Y, X-1}\right\rangle,\left|C_{Y-1, X-1}\right\rangle,\left|C_{Y, X}\right\rangle, \\
& \left|C_{Y-1, X}\right\rangle,\left|C_{Y-1, X+1}\right\rangle,\left|C_{Y, X+1}\right\rangle
\end{aligned}
$$

Step-3 The overall gradient $|G\rangle$ value of every pixel is estimated by finding the maximum pixels value in vertical, horizontal, $45^{0}$ and $135^{\circ}$ directions (more details in Supplementary S.3), and it can be represented as

$$
\frac{1}{2^{n}} \sum_{Y=0}^{2^{n}-1} \sum_{X=0}^{2^{n}-1}|M\rangle\left|G_{Y, X}^{i}\right\rangle|Y\rangle|X\rangle
$$

where $i=V, H, D_{1}, D ;|M\rangle,\left|G_{Y, X}^{i}\right\rangle=1$ for gradient values and $|M\rangle,\left|G_{Y, X}^{i}\right\rangle=0$ for out of gradient values.

Step-4 In this step, again cyclic shift transformation is used on the prepared nine quantum image set. All the shifting quantum image set of $3 \times 3$ neighborhood pixels are stored in ancillary qubits. The quantum arithmetic operations are performed according to the NMS operation (supplementary S.4). The output quantum states $\left|G^{N S}\right\rangle$ can be represented as

$\left|G^{N S}\right\rangle=\frac{1}{2^{n}} \sum_{Y=0}^{2^{n}-1} \sum_{X=0}^{2^{n}-1}|N\rangle\left|G_{Y X}\right\rangle|Y\rangle|X\rangle$

where $|N\rangle=1$ or 0 for maximum or non-maximum pixel value, respectively.

Step-5 Four quantum comparators (supplementary S.5) and quantum division circuit [28] are employed for comparing the gradient values in $0^{0}, 45^{\circ}, 90^{\circ}$, and $135^{\circ}$ directions using the adaptive threshold $\left|T_{H}\right\rangle$. The threshold value is determined from one third grayscale value of $3 \times 3$ neighborhood pixels window, and this threshold value is compared with each of the gradient pixel values in the four directions of $3 \times 3$ neighborhood pixels window. If the gradient value is greater than or equal to the threshold value, the edge point is obtained, otherwise no edge point and is carried out by an auxiliary qubit $|N\rangle$. Nine extra qubits are used to store the $3 \times 3$ neighborhood pixels window. The quantum states of the adaptive threshold $\left|T_{H}\right\rangle$ can be written as follows:

$$
\begin{aligned}
\left|T_{H}\right\rangle= & \left.\frac{1}{3} \times \frac{1}{9}|| C_{Y-1, X}\right\rangle+\left|C_{Y-1, X+1}\right\rangle+\left|C_{Y, X+1}\right\rangle \\
& +\left|C_{Y+1, X+1}\right\rangle+\left|C_{Y+1, X}\right\rangle+\left|C_{Y+1, X-1}\right\rangle \\
& +\left|C_{Y, X-1}\right\rangle+\left|C_{Y-1, X-1}\right\rangle+\left|C_{Y, X}\right\rangle \mid
\end{aligned}
$$

Step-6 The quantum state of the edge pixels can be written as

$$
\left|E_{D}\right\rangle=\frac{1}{2^{n}} \sum_{Y=0}^{2^{n}-1} \sum_{X=0}^{2^{n}-1}|N\rangle\left|E_{Y X}\right\rangle|Y\rangle|X\rangle ; E_{Y, X} \in\{0,1\}
$$

where $N, E_{Y, X}=0$ for non-edge point and $N, E_{Y, X}=1$ for edge point. The quantum circuit is shown in supplementary Fig-S.6.

\section{Analysis of Extracted Edges of CT Images}

Environment of computer:

\begin{tabular}{ll}
\hline Operating system & Windows 10 HSL(64 bit) \\
Image processing & MATLAB 9.4.0 (R2018a) \\
Chest CT image detail & $400 \times 400,8$-bit grayscale, jpg \\
Processor & Intel@Core ${ }^{\text {TMi }} 58300 \mathrm{HCPU} @ 2.30 \mathrm{GHz}$ \\
RAM & $8.00 \mathrm{~GB}$ \\
HDD Capacity & $1 \mathrm{~TB}$ \\
\hline
\end{tabular}

The NEQR method is employed to convert the grayscale images into quantum image representation with a size of $2^{n} \times$ 


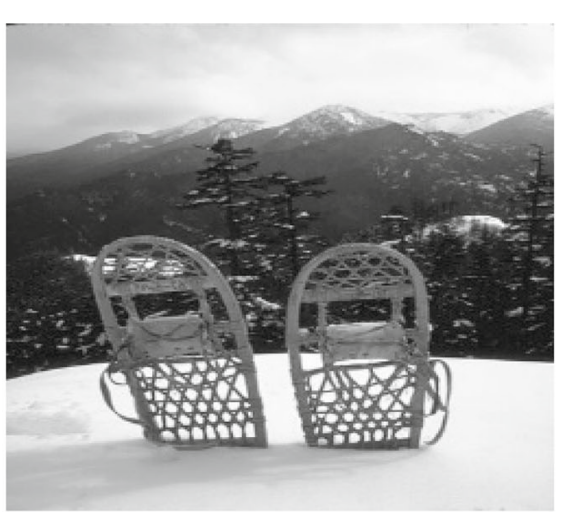

(i)

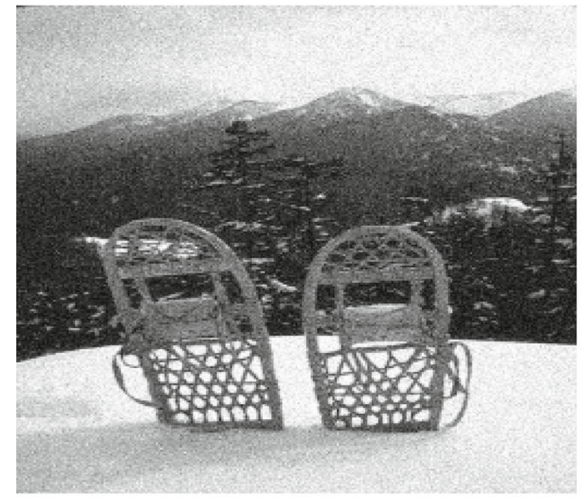

(iv)

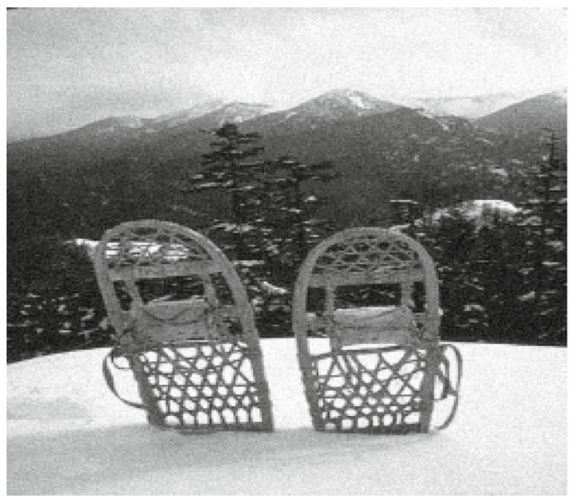

(ii)

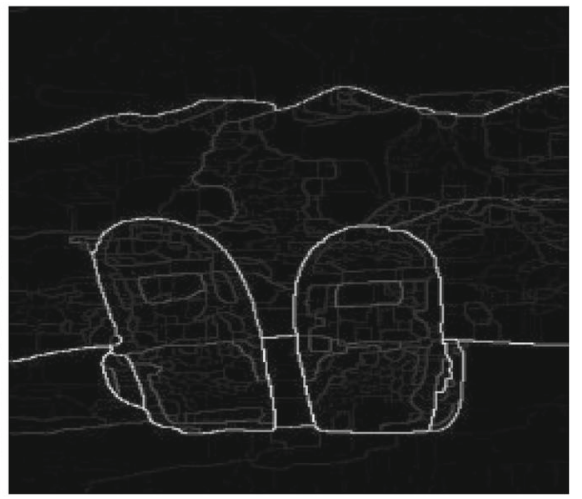

(v)

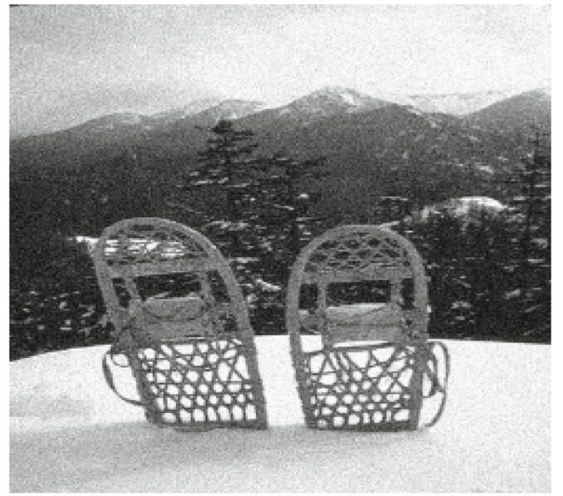

(iii)

Fig. 2 (i) Original image of 2018(BSDS500) [29]; (ii) noisy image (GN, mean $=0$, variance $=0.002)$; (iii) noisy image (GN, mean $=0$, variance $=0.004)$; (iv) noisy image $(\mathrm{GN}$, mean $=0$, variance $=0.006)$; (v) ground truth image of 2018(BSDS500) [29]

MSE

$\operatorname{PSNR}(\mathrm{dB})$

SSIM

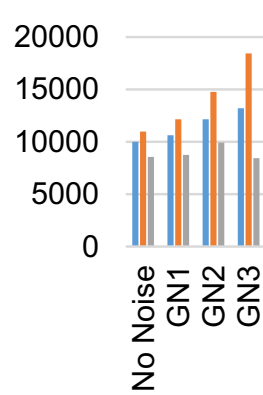

- Classical Sobel

- Quantum

Sobel

- Our algorithm

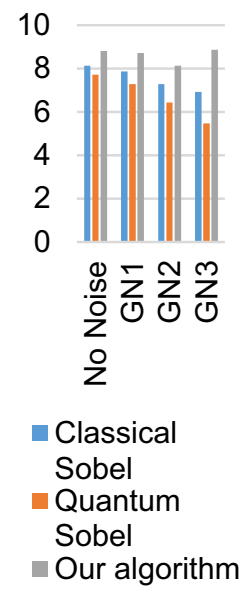

0.6

0.4

0.2

0 $\stackrel{\infty}{\frac{\infty}{0}} \sum_{i} \sum_{i}^{N} \sum_{0}^{m}$ ㄴ.

- Classical Sobel

- Quantum Sobel

- Our algorithm

Fig. 3 Graphical analysis of PSNR [30], MSE [30], and SSIM [28] of Fig. 4 for classical Sobel, quantum Sobel, and our edge detection algorithm (Supplementary-S 8: Table-S.8)

$2^{n}$. The simulations of our algorithm are based on linear algebra. The qubits (quantum states) are expressed as a complex vector, and the unitary matrices act as unitary transforms.

Before edge extraction of chest CT images, we need to compare the performance of our algorithm based on the adaptive threshold (as discussed earlier) in terms of structural similarity index measure (SSIM) [28], PSNR [30], and MSE [30] with respect to other algorithms with the standard threshold of value 128 [17, 25]. Here, we have computed edge extraction of 2018(BSDS500)[29] image(size $400 \times$ 400 ) having ground truth by using our algorithm and existing techniques for a different level of Gaussian noise (GN) deviates (Fig. 2) due to having CT images susceptible to Gaussian noise because of the appearance of electrical signals. Figure 3 shows that the PSNR and SSIM of the proposed method are higher than that of other algorithms (Supplementary-S 8: Table-S.8). The MSE values obtained using our algorithm are also significantly reduced in comparison with other existing techniques. The performance of the proposed algorithm has also been analyzed in the presence of Gaussian noise with variance $0.002,0.004$, and 0.006 showing less noise proportion than the other two existing edge extraction techniques (Fig. 4). For the comparison with recently reported works $[18,35]$, we have carried out edge extraction of standard Lena image using our algorithm (supplementary Fig-S.10.1) 

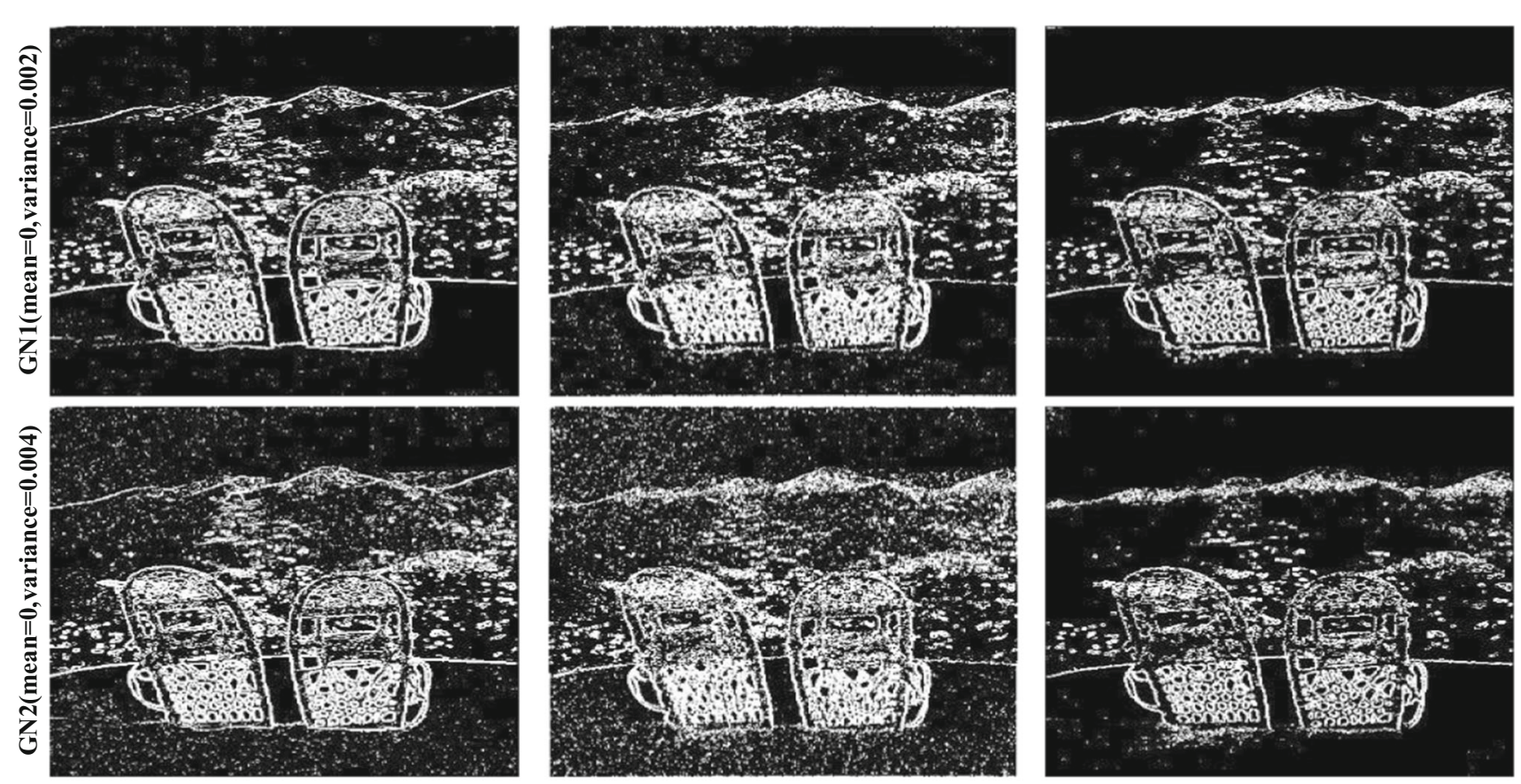

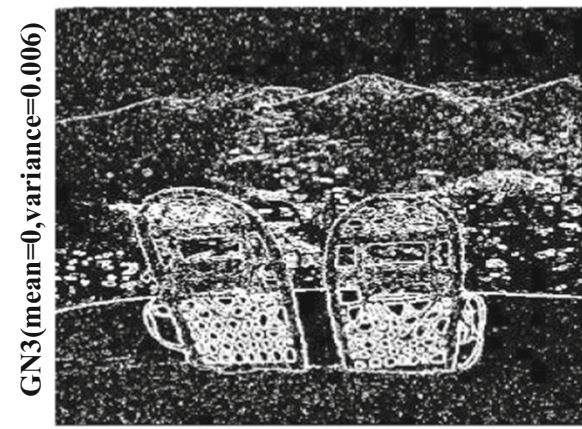

(a)

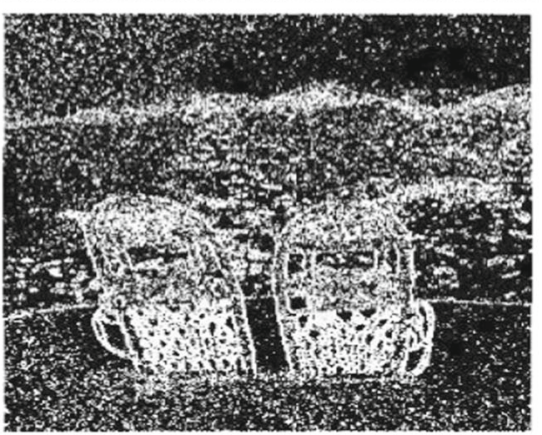

(b)

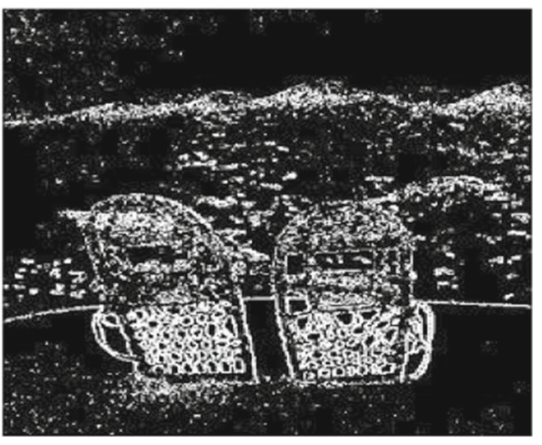

(c)

Fig. 4 a Classical Sobel edge extraction [17]; b quantum Sobel edge extraction [25]; c Our ISQEENSAT algorithm

exhibiting more PSNR of value 42.4057 (supplementary: S.10.1).

In the initial stage of COVID-19 patients, a chest CT image shows a GGO pattern, other patterns such as Crazy paving pattern (CPP) and consolidation have appeared as the day progresses. We have analyzed extracted edges of chest CT images of COVID patients having a wide range of age groups of 10 years to 74 years (admitted at different hospitals) using our ISQEENSAT algorithm. Table 1 shows the chest CT image dataset from which CT image samples were obtained to perform the proposed algorithm. We have determined entropy (EN) and the number of edge pixels (NoEP) of the extracted images for all three cases. The classical Sobel and quantum Sobel edge extracted images are shown in supplementary-S.8. It has been observed that the entropy [28] and NoEP of the extracted images using our proposed technique provide higher values compared to other existing methods [17, 21]. The proposed technique can detect the same clinical characteristics with more details about the contours even in the early stages of COVID-19 patients.

\subsection{Case-1}

CT imaging of COVID-19 patients of age groups of 31 years to 74 years was carried out at Taizhou Enze Hospital, China, from January 19, 2020, to February 4, 2020 [31]. The chest image of a 74-year-old male patient in Fig. 5a shows bilateral subpleural ground-glass opacities (GGO) with slight consolidation in the Lungs, and the corresponding clinical edges of COVID-19 in the lungs are extracted successfully by ISQEENSAT algorithm as shown in Fig. 5A. The chest image of a 43-year-old female patient in Fig. 5b represents subpleural GGO and slight consolidation in the lungs [31]. Here, clinical edges in the lungs are extracted by using the ISQEENSAT algorithm. Figure 5c reveals a single focal GGO in the right upper lobe of the lungs [31], and the extracted image (Fig. 5C) displays the same clinical features. 
Table 1 Dataset of chest CT image used for the edge extraction of our proposed algorithm

\begin{tabular}{|c|c|c|c|c|c|}
\hline References & Author & Article name & Task & Data type & Age group in years \\
\hline [11] & Pan et al. & $\begin{array}{l}\text { Time course of lung } \\
\text { changes at chest CT } \\
\text { during recovery from } \\
\text { Coronavirus Disease } \\
2019 \text { (COVID-19) }\end{array}$ & $\begin{array}{l}\text { Identification of changes } \\
\text { in chest CT results for } \\
\text { COVID-19 from initial } \\
\text { diagnosis to patient } \\
\text { recovery }\end{array}$ & Chest CT image & $25-63$ \\
\hline [31] & Fang et al. & $\begin{array}{l}\text { Sensitivity of chest CT } \\
\text { for COVID-19: } \\
\text { comparison to } \\
\text { RT-PCR. Radiology }\end{array}$ & $\begin{array}{l}\text { The COVID-19 } \\
\text { detection rate was } \\
\text { compared using initial } \\
\text { chest CT and RT-PCR } \\
\text { results }\end{array}$ & $\begin{array}{l}\text { Chest CT image, } \\
\text { RT-PCR test } \\
\text { performed within } \\
3 \text { days }\end{array}$ & $31-74$ \\
\hline [32] & Chen et al. & $\begin{array}{l}\text { Differences in clinical } \\
\text { and imaging } \\
\text { presentation of } \\
\text { pediatric patients with } \\
\text { COVID-19 in } \\
\text { comparison with adults }\end{array}$ & $\begin{array}{l}\text { Characterize and } \\
\text { compare the initial } \\
\text { clinical and imaging } \\
\text { features of COVID-19 } \\
\text { among pediatric and } \\
\text { adult chest CT patients }\end{array}$ & Chest CT image & 2 months -62 years \\
\hline $\begin{array}{l}\text { [33] } \\
\text { (Supplementary-S.9) }\end{array}$ & Chan et al. & $\begin{array}{l}\text { A familiar cluster of } \\
\text { pneumonia associated } \\
\text { with the } 2019 \text { novel } \\
\text { coronavirus indicating } \\
\text { person-to-person } \\
\text { transmission: a study } \\
\text { of a family cluster }\end{array}$ & $\begin{array}{l}\text { COVID-19 transmission } \\
\text { and detection }\end{array}$ & $\begin{array}{l}\text { Epidemiological, } \\
\text { clinical laboratory, } \\
\text { radiological (CT } \\
\text { image), and } \\
\text { microbiological }\end{array}$ & $10-66$ \\
\hline $\begin{array}{l}\text { [34] } \\
\text { (Supplementary-S.9) }\end{array}$ & Ajlan et al. & $\begin{array}{l}\text { Middle East Respiratory } \\
\text { Syndrome Coronavirus } \\
\text { (MERS-CoV) } \\
\text { infection: chest CT } \\
\text { findings }\end{array}$ & COVID-19 detection & Chest CT image & $27-83$ \\
\hline
\end{tabular}

In the case of a 31-year-old male patient with a one-day symptom, the CT image in Fig. 5d shows a mild linear GGO in the left lower lateral mid lung due to having less number of infected days, and similarly extracted image in Fig. 5D also displays mild linear clinical patterns. The extracted images show peripheral GGO patterns at the initial stage of infection, and so as the day progresses, the area of GGO patterns with consolidation increases, especially in the case of aged patients. The graphical analysis of entropy and NoEP shows that the proposed algorithm can extract more clinical information than other methods considered (Fig. 6). The images extracted in Fig. 5 show that the proposed technique can detect the same clinical features with more detail on the edge information. Edge extraction of existing conventional and quantum Sobel operations, entropy and NoEP difference are shown in Supplementary Figures S.8 and Table S.8.1, respectively.

\subsection{Case-2}

We have analyzed CT imaging of COVID-19 patients of age groups 28-54 years admitted at the Territorial medical unit of Guangzhou, China [32]. The chest image of 36-year-old female patient with a one-day symptom in Fig. 7a shows subpleural GGO in the lower portion of the left lobe of the lungs [32] and the corresponding clinical edges are extracted using our algorithm with minimal noise suppression located in the chest CT image, shown in Fig. 7A. The chest image of the male 54-year-old patient with 4-day symptom in Fig. 7b displays Subpleural ground-glass opacity in the lower portion of the left lobe and crazy paving pattern (inter-lobular and intra-lobular septal thickening) and a ground-glass nodule in the right lower lobe of lungs [32].

Here, clinical edges in the lungs are extracted using ISQEENSAT algorithm. The subpleural GGO in the left lower lobe with central consolidation and central GGO in the upper portion of the right lobe of lungs have appeared in CT image of a Male 28-year-old patient with 3-day symptom (Fig. 7c) [32] and the edges extracted in Fig. 7C by the algorithm displays same clinical features. The extracted image shows GGO patterns at the initial stage of infection of the patients. Like, case-1 as the day progresses, the area of GGO patterns increases, crazy paving pattern (CPP) and consolidation are enhanced, especially in aged patients. Supplementary Figure S.8.2 and Table S.8.2 show the difference in entropy and NoEP between existing conventional and quantum Sobel operations (Fig. 8). 


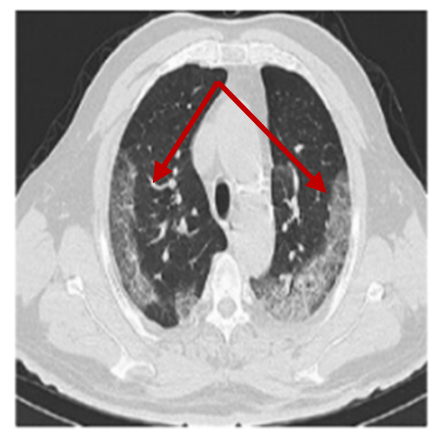

(a)

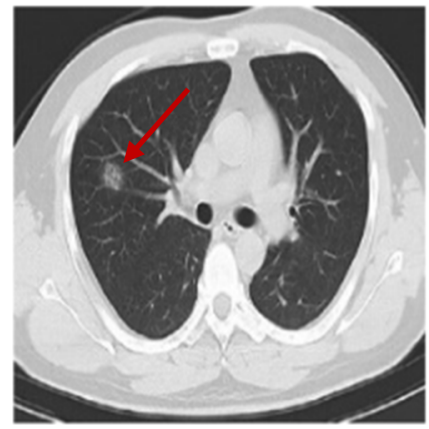

(c)

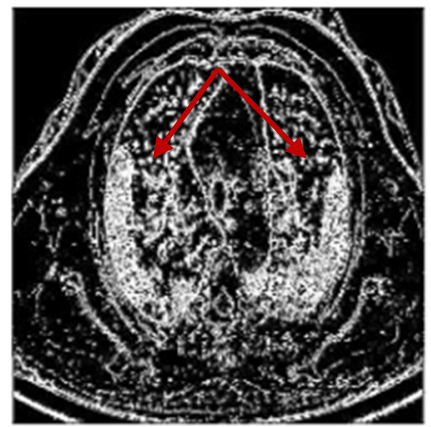

(A)

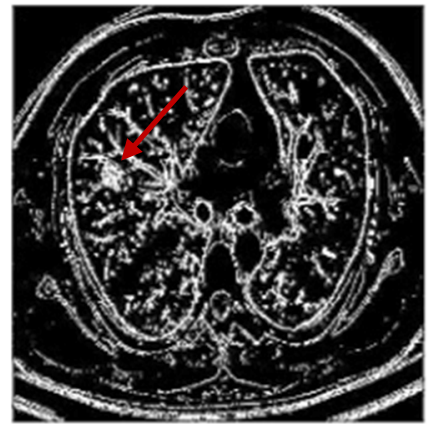

(C)

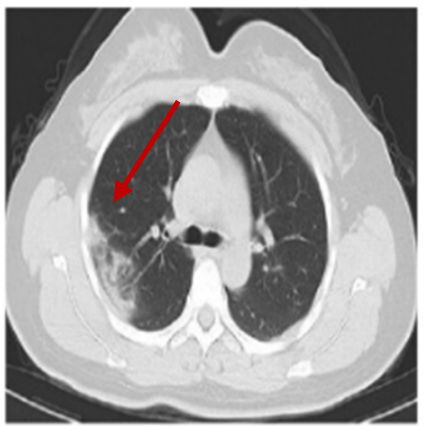

(b)

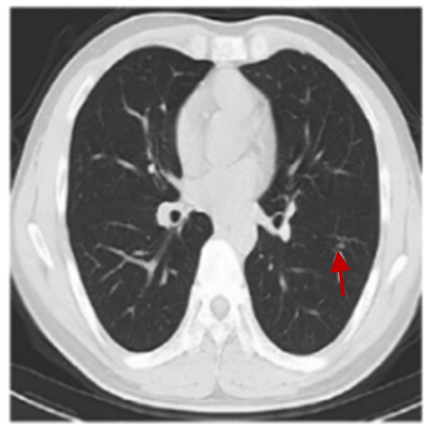

(d)

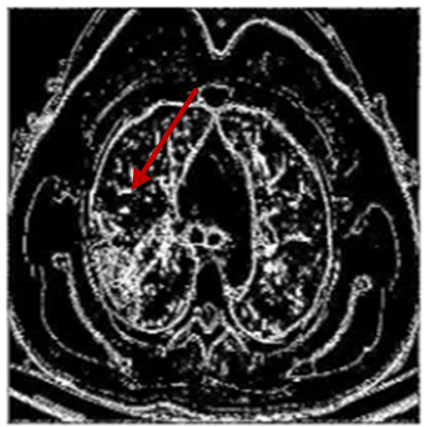

(B)

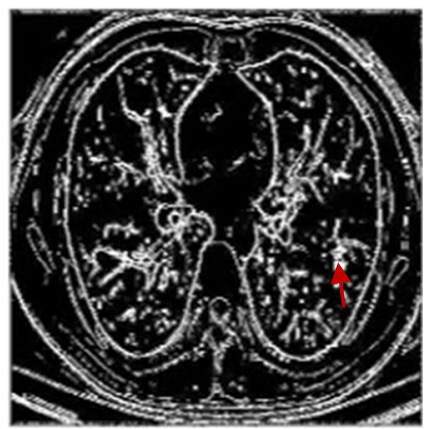

(D)

Fig. 5 Transaxial chest CT images [31] and edges of clinical patterns extracted by our ISQEENSAT algorithm: a Male 74-year-old patient with 5 days fever symptom: chest CT shows bilateral subpleural GGO A extracted edges of CT image of (a). b Female 43-year-old patient with 5 days fever symptom. Chest CT shows subpleural GGO and slight consolidation B extracted edges of CT image of (b). c Male 36-year-old

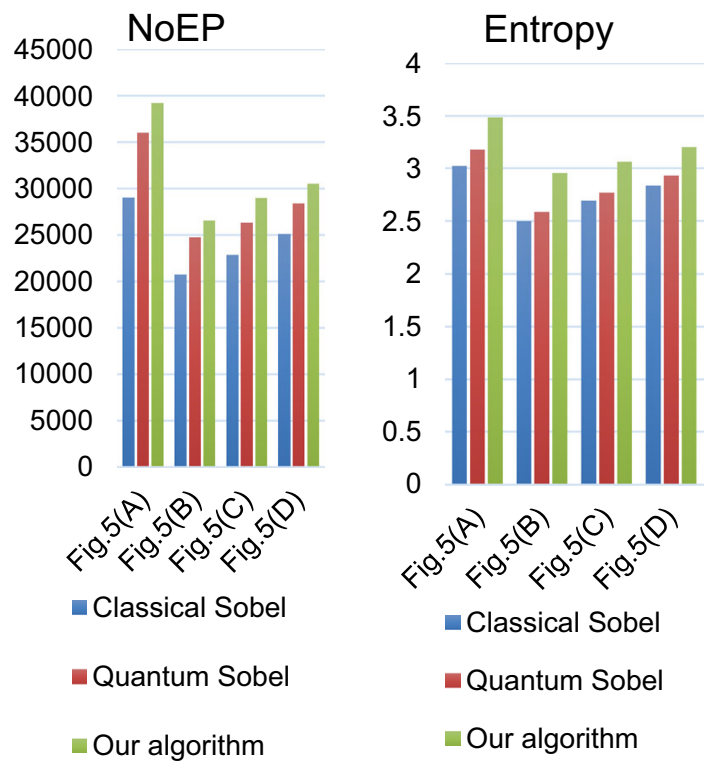

Fig. 6 Graphical analysis of entropy and no of edge pixels (NoEP) values for the extracted image of Fig. 5 (Supplementary-S8: Table-S.8.1)

patient with 3 days fever symptom having a small focal and central GGO in the right upper lobe $\mathbf{C}$ extracted edges of CT image of (c). d Male, 31 years old with fever for 1 day. Axial chest CT image shows a linear GGO in the left lower lateral mid lung. D Extracted edges of CT image of (d). Extracted images of classical Sobel and quantum Sobel are described in supplementary-S.8

\subsection{Case-3}

As per CT image analysis, normally, there are four stages of COVID-19 patients [11]—-stage-1 representing early-stage (0-4 days), stage-2 indicating progressive stage (5-8 days), stage- 3 showing peak stage (9-13 days), and stage-4 indicating absorption stage (14-21 days). We have employed our algorithm on CT images of different stages of COVID-19 patients [11] for the extraction of the clinical features. Figure 9A shows the appearance of edges location of peripheral GGO having distributed subpleural in the lower lobes unilaterally and bilaterally with partial consolidation on left lung in $75 \%$ patients extracted from CT image recorded in between 0 and 4 days (Fig. 9a) [11]. Figure 9B shows the appearance of sharp edges of GGO extended to more pulmonary lobe with CPP and consolidation on the left lung of $53 \%$ of patients extracted from CT image recorded in between 5 and 8 days (Fig. 9b) [11]. Figure 9C represents extracted sharp edges, especially in the smooth infected regions of consolidation residual parenchymal bands with diffused GGO and CPP in the left lung from CT images (Fig. 9c) carried out between 9 and 13 days. Figure 9D represents edges of consolidation 


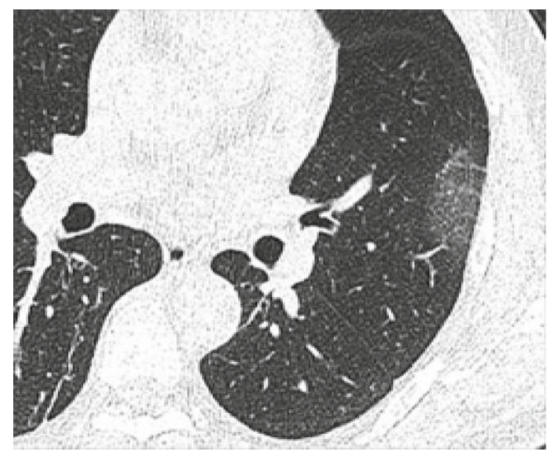

(a)

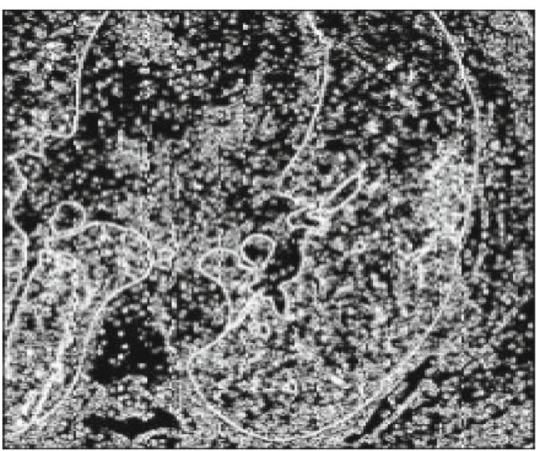

(A)

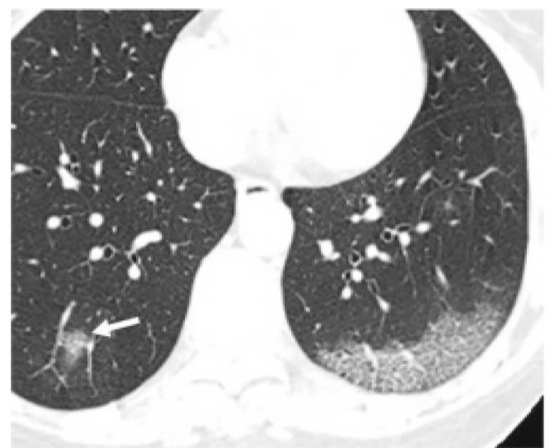

(b)

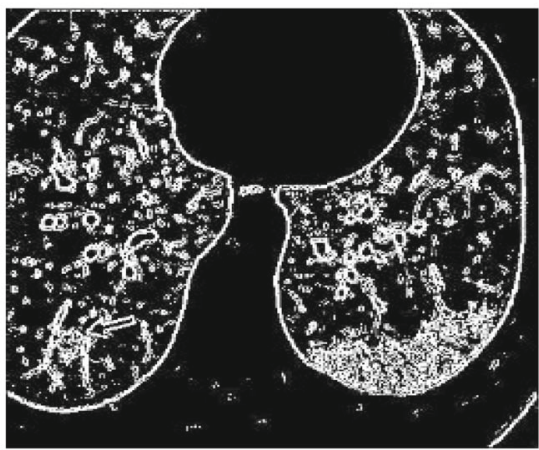

(B)

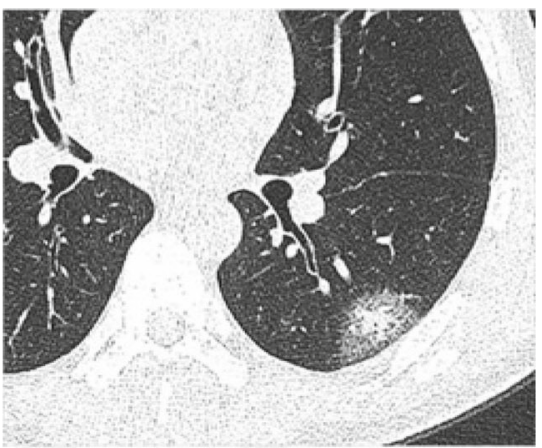

(c)

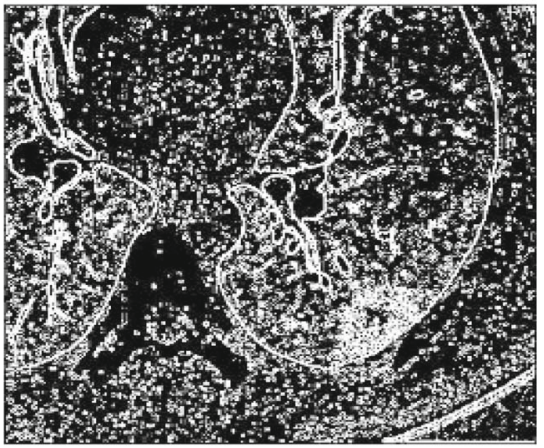

(C)
Fig. 7 Transaxial chest CT scanned images [32] and edges of clinical patterns extracted by our ISQEENSAT algorithm. a Female 36-year-old patient with 1-day symptom: chest CT shows subpleural GGO in left lower lobe; A extracted edges of CT image of (a). b Male 54-year-old patient with 4-day symptom. Chest CT shows subpleural ground-glass opacity in left lower lobe with CPP (inter-lobular and intralobular sep- tal thickening) and a ground-glass nodule in the right lower lobe $\mathbf{B}$ extracted edges of CT image of (b). (c) Male 28-year-old patient with 3-day symptom; CT image reveals subpleural ground-glass opacity in the left lower lobe with central consolidation $\mathbf{C}$ extracted edges of CT image of (c). Extracted images of classical Sobel and quantum Sobel are described in supplementary-S.8

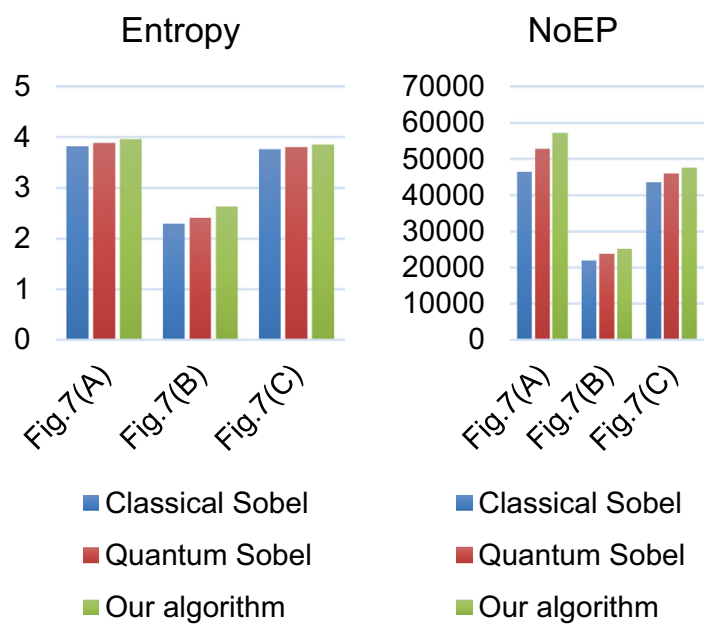

Fig. 8 Graphical analysis of Entropy and no of edge pixels (NoEP) values (Supplementary-S8: Table-S.8.2) for the extracted image of Fig. 7

bands (partially absorbed) having a slight appearance of CPP in the left lung extracted from CT images (carried out during 14-21 days).
It is evident from extracted images in Fig. $9 \mathrm{~A}-\mathrm{C}$ as the day progresses, the infected area in the lungs increases and becomes maximum at the peak stage. In the absorption stage, the infected area decreases due to absorption of consolidation and CPP (Fig. 9D). The proposed technique extracted the edges of the smooth region very clearly. Moreover, the graphical analysis of entropy [28] and NoEP difference values provide better performance compared to Sobel's classical [17] and quantum operators [25] (Supplementary Table S.10). The extracted images of Fig. 9 using classical Sobel operator and quantum Sobel operator are shown in supplementary- Fig. S.10. Analysis of the edge extraction of chest $\mathrm{CT}$ images assures the infected region of the different stages of COVID-19 patients after admitting to the hospital and recovery from the infections (Fig. 10).

\section{Results and Discussion}

Although novel coronavirus nucleic acid detection through rRT-PCR is a standard testing reference accepted all over 


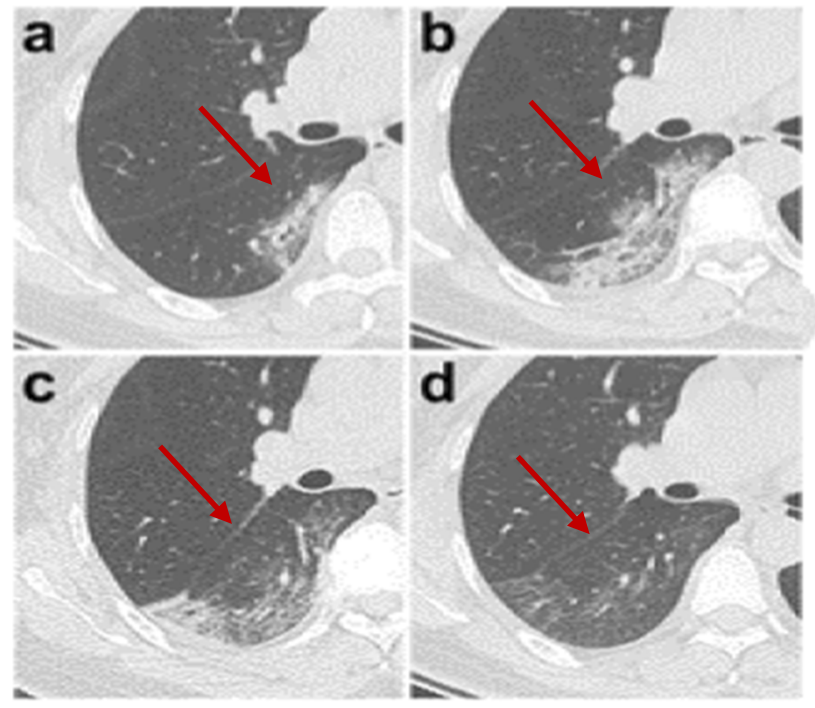

Fig. 9 Original CT scanned image for different stages of COVID-19 of 47-year-old woman[11]; a early-stage CT image; b progressive stage CT image; c peak stage CT image; $\mathbf{d}$ absorption stage CT image. A Extracted image of early stage using our algorithm. B Extracted image

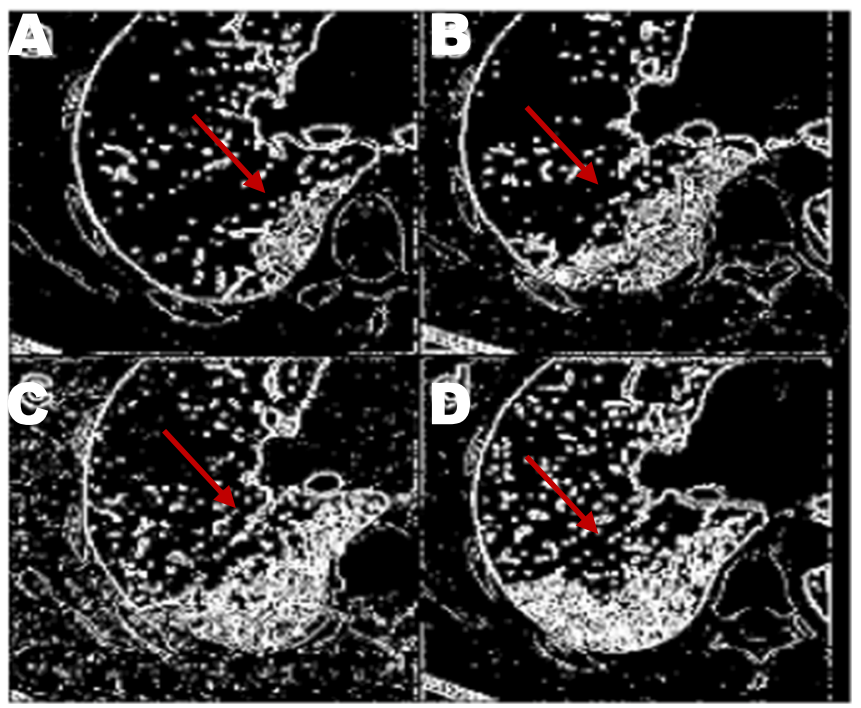

of progressive stage using our algorithm. C Extracted image of peak stage using our algorithm. D Extracted image of absorption stage using our algorithm

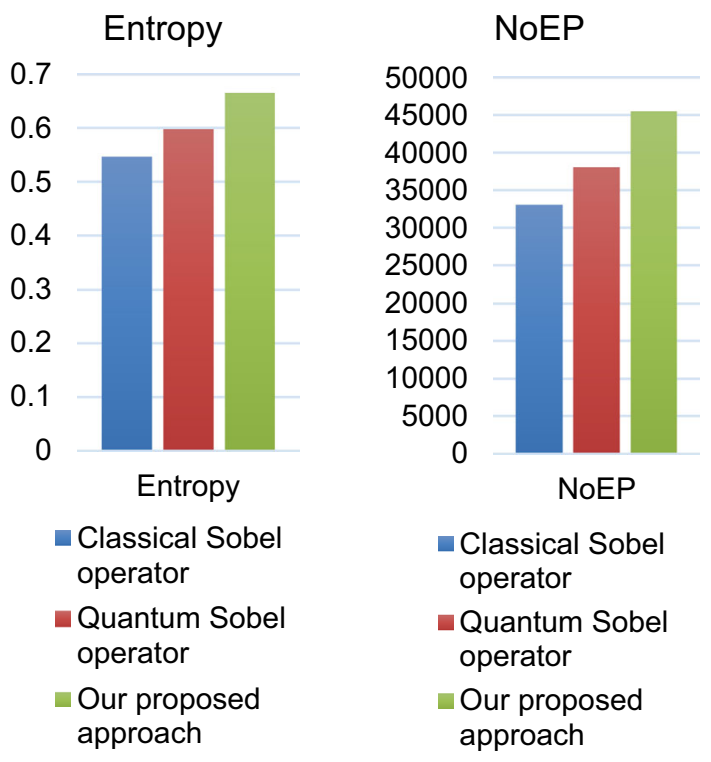

Fig. 10 Graphical analysis of Fig. 9 (Supplementary-S.10: Table-S.10): a entropy difference values. $\mathbf{b}$ NoEP of the extracted images

the world, the rate of false negatives is also high because of the nasopharyngeal swab sampling error requiring repeated samples [31]. Moreover, due to the time-consuming and expensive nature of rRT-PCR testing, delayed isolation and treatment may cause the spread of the disease through human-to-human transmission in highly dense populated countries such as India, Bangladesh, UK, and France, Edge extraction from high-resolution CT images can be used for early detection and analysis of COVID-19.
After studying CT images of the patients of different ages in different regions of the World and their edge extracted images indicating clinical lesions/patterns. Fig-5D and Fig7A show that in the initial stage of COVID-19 patients, GGO patterns appear either in the left or right lobe of the lungs. In later stages, the lungs are affected more by a novel coronavirus, and consequently, GGO with consolidation and CPP have appeared with the impact management of the patients, as shown in Figs. 7B, 9B, C. The CT images of pediatric patients indicate lesser area of GGO, CPP, and consolidation compared to other patients in the age group. (Supplementary Fig. S.9 b).

Chest CT images differ from infection with other coronaviruses such as SARS-CoV [3,4] and MERS-CoV [34]. The early pathologic finding in the lungs infected with COVID19 was diffuse alveolar damage [31]. The exudation and edema in the alveoli cell of lungs possibly cause GGO on CT image in the early stage of infection of COVID-19. The enlarged mediastinal and hilar lymph nodes, pleural effusion, and pleural thickening, are not normally appeared in the early stages of COVID-19 infected lungs. Figures 5A, 9A, B show that the proposed technique can extract and differentiate these clinical patterns that have been observed in the later stages of COVID-19 infection. Normally GGO appears in the periphery of the lungs of COVID-19 patients at early stages (Fig. 9A). The CT and edge extraction images in Supplementary-S.9: Figure S.9a and S.9b of MERS-COVinfected patients shows minimal reticulation, per lobular ground-glass opacities, and extensive ground-glass opacities [34], whereas in supplementary Figure S.9B, patients 
infected with SERS-COV- [33] show the multi-focal GGO patterns $[3,4]$. We have also extracted edges of chest CT images of the above cases of COVID-19 by using classical Sobel operator [17] and quantum Sobel operator without non-maximum suppression (supplementary-S.8: Fig.S.8, and Fig. S.10).We have estimated entropy (EN) and the number of edge pixels (NoEP) of the extracted images for all three cases. The classical Sobel and quantum Sobel edge extracted images are shown in supplementary-S.8 and S.10. It has been observed that the extracted image of our proposed technique provides a higher entropy and edge information compared to other existing methods [17, 25]. The ISQEENSAT algorithm extracts more sharp edges of the clinical pattern than that using the classical Sobel operator [17] and quantum Sobel operator [21, 25]. This is due to the use of four directions (vertical, horizontal, and two diagonal) extraction of edges in our algorithm, whereas in the case of classical Sobel operator and quantum Sobel operator, only vertical extraction and horizontal extraction are employed. The extracted edges carrying clinical information are sharper and can suppress minimal noise in the chest CT image than the existing Sobel operators $[17,21,25]$ due to the use of non-maximum suppression and adaptive threshold. The extracted sharp edges even in the smooth regions representing clinical lesions of infected lungs. The proposed technique can extract the clinical information from the smooth, low-intensity boundary region, and it can be a useful practice for the early diagnosis of COVID-19 detection.

Generally, the circuit complexity of quantum image preparation, the measuring processes are not taken into consideration in the quantum image algorithm. The overall circuit complexity of our ISQEENSAT algorithm is written as $\sim \mathrm{O}$ $\left(n^{2}+q^{3}\right)$, which provides exponential speedup (Supplementary S.7).

\section{Conclusion}

The rapid global outbreak of 2019-nCoV signals a very fast screening of COVID-19 infected patients for isolation and quarantine. Here, our ISQEENSAT algorithm has been applied on Chest CT images of infected patients for extraction of sharp edges of clinical features of lunges with minimal suppression of noises located at the chest. The edge extracted images of CT show GGO appeared at the periphery of the lungs, indicating initial stages of COVID-19 patients, and thickened peripheral GGO with CPP and consolidation are observed in later stages of infection. The result of CT extracted images assures a fast noninvasive screening, diagnosis, and detection of the infected region of COVID-19 patients without risk of infection. Moreover, the proposed algorithm shows a better performance with less noise proportion compares to the other two existing edge extraction techniques in the presence of Gaussian noise. In particular, the proposed technology has very clearly extracted the edges of the smooth area. The application of the ISQEENSAT technique with quantum denoising filter on different types of medical imaging for the detection of the region of other diseases is one of the future works.

Supplementary Information The online version contains supplementary material available at https://doi.org/10.1007/s13369-021-06511-9.

Acknowledgements The authors are grateful to Dr. Somen Misra, MS of All India Institute of Medical Science, Raipur, for clinical analysis of CT image and its extracted images. The authors are also grateful to Mr. S. M, B. Baruah, Department of ECE, Tezpur University, for his help in developing MATLAB code.

\section{Declarations}

Conflict of interest Authors declare that there is no conflict of interest.

Human and Animal Rights This article does not contain any studies with human participants performed by any of the authors.

\section{References}

1. Huang, C.; Wang, Y., et al.: Clinical features of patients infected with 2019 novel coronavirus in Wuhan, China. Lancet 395(10223), 497-506 (2020)

2. Cheng, S.C.; Chang, Y.C., et al.: First case of Coronavirus Disease 2019 (COVID-19) pneumonia in Taiwan. J. Formosan Med. Assoc. 119, 747-751 (2020)

3. Yeung, M.C.; Xu, R.H.: SARS: epidemiology. Respirology 8, S9-S14 (2003)

4. Andersen, K.G.; Rambaut, A.; Lipkin, W.I.; Holmes, E.C.; Garry, R.F.: The proximal origin of SARS-CoV-2. Nat. Med. 26, 450-452 (2020)

5. Pitman, R.J.; Cooper, B.S.; Trotter, C.L.; Gay, N.J.; Edmunds, W.J.: Entry screening for severe acute respiratory syndrome (SARS) or influenza: policy evaluation. BMJ 331(7527), 1242-1243 (2005)

6. Lee, V.J.; Chiew, C.J.; Khong, W.X.: Interrupting transmission of COVID-19: lessons from containment efforts in Singapore. J. Travel Med. 27(3), taaa09 (2020)

7. Zhang, Y.; Xiao, M.; Zhang, S.; Xia, P.; Cao, W.; Jiang, W.; Chen, H.; Ding, X.; Zhao, H.; Zhang, H.; Wang, C.; Zhao, J.; Sun, X.; Tian, R.; Wu, W.; Wu, D.; Ma, J.; Chen, Y.; Zhang, D.; Xie, J.; Yan, X.; Zhou, X.; Liu, Z.; Wang, J.; Du, B.; Qin, Y.; Gao, P.; Qin, X.; Xu, Y.; Zhang, W.; Li, T.; Zhang, F.; Zhao, Y.; Li, Y.; Zhang, S.: Coagulopathy and antiphospholipid antibodies in patients with Covid-19. N. Engl. J. Med. 382, 17-38 (2020)

8. Wang, Y., et al.: Unobtrusive and automatic classification of multiple people's abnormal respiratory patterns in real time using deep neural network and depth camera. IEEE Internet Things J. 7(9), 8559-8571 (2020). https://doi.org/10.1109/JIOT.2020.2991456

9. Kanne, J.P.; Little, B.P.; Chung, J.H.; Elicker, B.M.; Ketai, L.H.: Essentials for radiologists on COVID-19: an update-Radiology Scientific Expert Panel. Radiology 296(2), E113-E114 (2020)

10. Han, R.; Huang, L.; Jiang, H.; Dong, J.; Peng, H.; Zhang, D.: Early clinical and CT manifestations of coronavirus disease 2019 (COVID-19) pneumonia. Am. J. Roentgenol. 215(2), 338-343 (2020) 
11. Pan, F.; Ye, T.; Sun, P.; Gui, S.; Liang, B.; Li, L.; Zheng, D.; Wang, J.; Hesketh, R.L.; Yang, L.; Zheng, C.: Time course of lung changes at chest $\mathrm{CT}$ during recovery from coronavirus disease 2019 (COVID-19). Radiology 295(3), 715-721 (2020). https://doi.org/ 10.1148/radiol.2020200370

12. Cutillo, A.G.; Goodrich, K.C.; Ganesan, K.; Watanabe, S.; Ailion, D.C.; Morris, A.H., et al.: Alveolar air/tissue interface and nuclear magnetic resonance behavior of normal and edematous lungs. Am. J. Respir. Crit. Care Med. 151(4), 1018-1026 (1995)

13. Li, Y.; Xia, L.: Coronavirus disease 2019 (COVID-19): role of chest CT in diagnosis and management. Am. J. Roentgenol. 214(6), 1280-1286 (2020)

14. Rasheed, J.; Hameed, A.A.; Djeddi, C.; Jamil, A.; Al-Turjman, F.: A machine learning-based framework for diagnosis of COVID-19 from chest X-ray images. Interdiscip. Sci. Comput. Life Sci. 13(1), 103-117 (2021)

15. Rasheed, J.; Jamil, A.; Hameed, A.A.; Aftab, U.; Aftab, J.; Shah, S.A.; Draheim, D.: A survey on artificial intelligence approaches in supporting frontline workers and decision makers for COVID-19 pandemic. Chaos Solitons Fractals 14, 110337 (2020)

16. Mydukuri, R.V.; Kallam, S.; Patan, R.; Al-Turjman, F.; Ramachandran, M.: Deming least square regressed feature selection and Gaussian neuro-fuzzy multi-layered data classifier for early COVID prediction. Expert Syst. (2021). https://doi.org/10.1111/exsy.12694

17. Kanopoulos, N., et al.: Design of an image edge detection filter using the Sobel operator. IEEE J. Solid State Circuits 23(2), 358-367 (1988)

18. Wang, H.; Zhou, G.; Zhang, Y.: Research on edge detection and thinning algorithm of improved Prewitt about dynamic threshold. Coal Technol. 28, 144-145 (2009)

19. Kimmel, R.; Bruckstein, A.M.: Regularized Laplacian zero crossings as optimal edge integrators. Int. J. Comput. Vis. 53(3), 225-243 (2003)

20. Canny, J.: A computational approach to edge detection. IEEE Trans. Pattern Artif. Mach. Intell. 8(6), 679-698 (1986)

21. Zhang, Y.; Lu, K.; Gao, Y.: QSobel: a novel quantum image edge extraction algorithm. Sci. China Inf. Sci. 58(1), 1-13 (2015)

22. Venegas-Andraca, S. E.; \& Bose, S.: Storing, processing, and retrieving an image using quantum mechanics. In: Quantum Information and Computation, vol. 5105, pp. 137-147. International Society for Optics and Photonics (2003)

23. Chetia, R.; Boruah, S.M.B.; Sahu, P.P.: Quantum image edge detection using improved Sobel mask based on NEQR. Quantum Inf. Process. 20(1), 1-25 (2021)

24. Chetia, R.; Boruah, S.M.B.; Roy, S.; Sahu, P.: Quantum image edge detection based on four directional Sobel operator. In: Deka, B.; Maji, P.; Mitra, S.; Bhattacharyya, D.; Bora, P.; Pal, S. (Eds.) Pattern Recognition and Machine Intelligence. PReMI 2019. Lecture Notes in Computer Science, Vol. 11941, pp. 532-540. Springer, Cham (2019). https://doi.org/10.1007/978-3-030-34869-4_58
25. Fan, P.; Zhou, R.G.; Hu, W.; Jing, N.: Quantum image edge extraction based on classical Sobel operator for NEQR. Quantum Inf. Process. 18(1), 1-23 (2019)

26. Yan, F.; Iliyasu, A.M.; Le, P.Q.: Quantum image processing: a review of advances in its security technologies. Int. J. Quantum Inf. 15(3), 1730001 (2017)

27. Zhang, Y.; Lu, K.; Gao, Y.; Wang, M.: NEQR: a novel enhanced quantum representation of digital images. Quantum Inf. Process. 12(8), 2833-2860 (2013)

28. Iliyasu, A.M.; Yan, F.; Hirota, K.: Metric for estimating congruity between quantum images. Entropy 18(10), 360 (2016)

29. Arbelaez, P.; Maire, M.; Fowlkes, C.; Malik, J.: Contour detection and hierarchical image segmentation. IEEE Trans. Pattern Anal. Mach. Intell. 33(5), 898-916 (2010)

30. Bharodiya, A.K.; Gonsai, A.M.: An improved edge detection algorithm for X-ray images based on the statistical range. Heliyon 5(10), e02743 (2019)

31. Fang, Y.; Zhang, H.; Xie, J.; Lin, M.; Ying, L.; Pang, P.; Ji, W.: Sensitivity of chest CT for COVID-19: comparison to RT-PCR. Radiology 296(2), E115-E117 (2020)

32. Chen, A.; Huang, J.X.; Liao, Y.; Liu, Z.; Chen, D.; Yang, C.; Yang, R.; Wei, X.: Differences in clinical and imaging presentation of pediatric patients with COVID-19 in comparison with adults. Radiol. Cardiothorac Imaging 2(2), e200117 (2020)

33. Chan, J.F.W.; Yuan, S.; Kok, K.H.; To, K.K.W.; Chu, H.; Yang, J.; Xing, F.; Liu, J.; Yip, C.C.-Y.; Poon, R.W.-S.; Tsoi, H.-W.; Lo, S.K.-F.; Chan, K.-H.; Poon, V.K.-M.; Chan, W.-M.; Ip, J.D.; Cai, J.P.; Cheng, V.C.-C.; Chen, H.; Hui, C.K.-M.; Yuen, K.Y.: A familial cluster of pneumonia associated with the 2019 novel coronavirus indicating person-to-person transmission: a study of a family cluster. Lancet 395(10223), 514-523 (2020)

34. Ajlan, A.M.; Ahyad, R.A.; Jamjoom, L.G.; Alharthy, A.; Madani, T.A.: Middle East respiratory syndrome coronavirus (MERS-CoV) infection: chest CT findings. Am. J. Roentgenol. 203(4), 782-787 (2014)

35. Ma, Y.; Ma, H.; Chu, P.: Demonstration of quantum image edge extration enhancement through improved Sobel operator. IEEE Access 8, 210277-210285 (2020) 